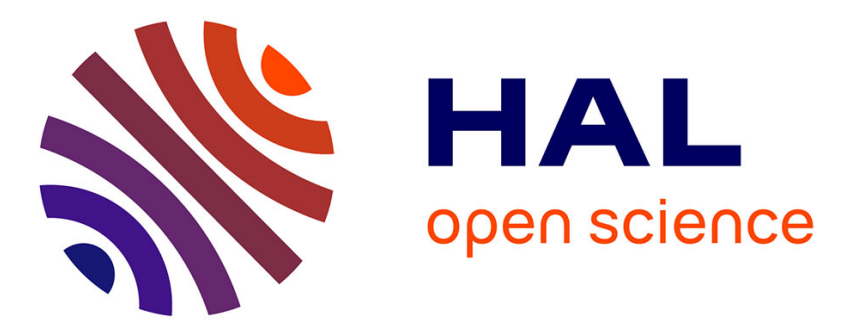

\title{
Passivity-based nonlinear control of CSTR via asymptotic observers.
}

N.H. Hoang, Françoise Couenne, Yann Le Gorrec, C.L. Chen, B. Erik Ydstie

\section{To cite this version:}

N.H. Hoang, Françoise Couenne, Yann Le Gorrec, C.L. Chen, B. Erik Ydstie. Passivity-based nonlinear control of CSTR via asymptotic observers.. Annual Review on Control, 2013, 37 (2), pp.1-33. 10.1016/j.arcontrol.2013.09.007 . hal-00876673

\section{HAL Id: hal-00876673 https://hal.science/hal-00876673}

Submitted on 25 Oct 2013

HAL is a multi-disciplinary open access archive for the deposit and dissemination of scientific research documents, whether they are published or not. The documents may come from teaching and research institutions in France or abroad, or from public or private research centers.
L'archive ouverte pluridisciplinaire HAL, est destinée au dépôt et à la diffusion de documents scientifiques de niveau recherche, publiés ou non, émanant des établissements d'enseignement et de recherche français ou étrangers, des laboratoires publics ou privés. 


\title{
Passivity-based nonlinear control of CSTR via asymptotic observers ${ }^{1}$
}

\author{
N. Ha Hoang ${ }^{\star}, \mathrm{a}$, F. Couenne ${ }^{\mathrm{b}}$, Y. Le Gorrec ${ }^{\mathrm{c}}$, C. L. Chen ${ }^{\mathrm{d}}$, B. Erik Ydstie \\ ${ }^{a}$ Faculty of Chemical Engineering, University of Technology, VNU-HCM, 268 Ly Thuong \\ Kiet Str., Dist. 10, HCM City, Vietnam \\ (e-mail: ha.hoang@hcmut.edu.vn) \\ ${ }^{b}$ LAGEP, University of Lyon, University of Lyon 1, UMR CNRS 5007, Villeurbanne, \\ France (e-mail: couenne@lagep.univ-lyon1.fr) \\ ${ }^{c}$ ENSMM Besançon FEMTO-ST / AS2M, Besançon, France (e-mail: \\ yann.le.gorrec@ens2m.fr) \\ ${ }^{d}$ Department of Chemical Engineering, National Taiwan University, Taiwan (e-mail: \\ ccl@ntu.edu.tw) \\ ${ }^{e}$ Chemical Engineering Dept., Carnegie Mellon University, 5000 Forbes Avenue, \\ Pittsburgh, PA 15213,USA (e-mail: ydstie@cmu.edu)
}

\begin{abstract}
This work makes use of a passivity-based approach (PBA) and tools from Lyapunov theory to design a nonlinear controller for the asymptotic stabilization of a class of non isothermal Continuous Stirred Tank Reactors (CSTR) around any desired stationary point. The convergence and stability proofs are derived in the port Hamiltonian framework. Asymptotic observers that do not require knowledge of reaction kinetics are also proposed for a system with incomplete state measurement. Numerical simulations are given to illustrate the application of the theoretical results to a CSTR with multiple steady states.
\end{abstract}

Keywords: CSTR, Asymptotic observers, Hamiltonian systems, Nonlinear control, Lyapunov function, Passivity.

\footnotetext{
${ }^{1}$ This work is an expanded version of the paper presented at the IFAC-International Symposium on Advanced Control of Chemical Processes, 10-13 July 2012, Singapore.

*Also CESAME, Université catholique de Louvain, 4-6 avenue G. Lemaître, B-1348 Louvain-la-Neuve, Belgium.
} 


\section{Introduction}

Lyapunov theory [1], or more generally the Passivity Based Approach (PBA) $[2,3,4]$ combined with generalized energetic arguments as expressed through a Hamiltonian function, is one of the most efficient ways to investigate stability and design controllers for nonlinear dynamical systems [5, 6, 7]. The key idea of the PBA in the Port Hamiltonian framework [8] is to define transformations (by means of control input or shaped dynamics) to obtain a certain structured representation of the original system by rendering it passive with respect to a given storage function. The PBA was first proposed and successfully applied for stability analysis and control design for the electromechanical systems $[9,10]$. In these systems the connections between the energy and the dynamical behaviour of the system are well established by the fact that the system reaches its stable state if and only if the total energy is at its minimum. As a consequence, a Lyapunov function candidate can be assigned to the total energy and passivity can then be related to energy dissipation due to friction or resistance. Unfortunately, the link between Lyapunov stability theory and the energy of chemical reactive systems is far from being understood at present $[11,12]$. This topic has therefore been an active research area $[13,14,15,16]$.

The Continuous Stirred Tank Reactors (CSTRs) [17] provide a benchmark both in chemical engineering and in dynamical systems theory due to their highly nonlinear dynamics. CSTRs may exhibit non-minimum phase behaviour [18], instability and multiple steady states [19, 12]. Studies on CSTRs have investigated control synthesis for stabilization $[20,21,15,23,11]$ and state observer design $[24,25,26,27]$. The combination of these is an important field of research.

The underlying motivation for nonlinear control of the CSTRs is that industrial chemical reactors may have to be operated at unstable operating conditions [28]. Numerous control strategies have been developed to achieve this objective. Input/output feedback linearization [19] for control under constraints, nonlinear PI control [29], direct Lyapunov based control [30], (pseudo) Hamiltonian framework [13, 14, 31, 21], power/energy-shaping control $[12,11]$, inventory control [32] and dissipativity based decentralized control of interconnected chemical reactors [33, 16] provide some examples. Thermodynamics/physics based control has also been proposed to the stabilization of chemical reactors in [20,34] and more recently in [15] using the availability function as its point of departure. 
State estimation for CSTRs has attracted the attention of researchers for a long time. Papers $[35,36]$ and references therein provide good overviews of recent developments. Strategies have been developed for industrial applications since on-line measurements of all reactant concentrations are difficult and/or quite expensive to implement and the reactor temperature is in some cases the only measurement available online [24, 26]. The missing state variables can be estimated by different tools [24, 25, 26, 37, 27]. The results given in the papers referred above relate to systems where feedback is not imposed. Closed loop stability can therefore not be guaranteed in general.

In this work we focus on the combined control and state estimation problems. First, we propose a passive nonlinear controller for the stabilization of the fully actuated CSTR with chemical reactions around a steady state which may be unstable. This approach is based on the passive Hamiltonian concepts defined in $[4,3,10]$. The shaped Hamiltonian storage function is chosen by using the techniques in $[19,32,15]$ such that the resulting state feedback is admissible [15]. Second, we assume that only the reactor temperature and a subset of concentrations are available online. Following the same concepts used for the passivity-based control, we propose a state estimation strategy based on chemical reaction invariants via the so-called asymptotic observers $[37,27]$. We show, analytically and/or with simulations, that exponential convergence of the estimated state variables and closed loop stability of the CSTR are guaranteed.

This paper is organized as follows. The passivity based approach is introduced and the state feedback control law is derived in section 2. The dynamical model of the CSTR case study is presented and preliminary results are presented in section 3. Section 4 is devoted to the design of a passive nonlinear controller within the port Hamiltonian framework. It is shown that the resulting control is asymptotically stable and admissible in terms of the amplitude and variation rate as long as the chosen closed loop Hamiltonian function is appropriate. The results generalize previous ones [19] without constraint on control input. Furthermore, they allow to rewrite the closed loop system dynamics into a port Hamiltonian representation. A state reconstruction method is then proposed via the so-called asymptotic observers $[37,27]$. The theoretical developments are then illustrated by simulation studies reported in section 5 . Conclusions and future perspectives of the work are given in section 6 . 


\section{The Passivity Based Approach (PBA)}

Let us consider nonlinear systems that are affine in the control input $u$ and whose dynamics is given by the following set of ordinary differential equations (ODEs) [1]:

$$
\frac{d x}{d t}=f(x)+g(x) u
$$

where $x=x(t) \in \mathbb{R}^{n}$ is the state vector, $f(x) \in \mathbb{R}^{n}$ is a smooth nonlinear function with respect to $x, g(x) \in \mathbb{R}^{n \times m}$ is the input-state map and $u \in \mathbb{R}^{m}$ is the control input.

The purpose of the PBA is to find a static state-feedback control $u=\beta(x)$ such that the closed loop dynamics becomes a dissipative Port Controlled Hamiltonian $(\mathrm{PCH})$ system $[10,8]$. The dynamics can then be written:

$$
\frac{d x}{d t}=Q_{d}(x) \frac{\partial \mathcal{H}_{d}(x)}{\partial x}
$$

where the controlled Hamiltonian storage function $\mathcal{H}_{d}(x)$ has a strict local minimum at the desired equilibrium $x_{d}$; and $Q_{d}(x)=\left[J_{d}(x)-R_{d}(x)\right]$ is the difference of a skew-symmetric matrix $J_{d}(x)$ and a symmetric one $R_{d}(x)$ so that:

$$
J_{d}(x)=\frac{Q_{d}(x)-Q_{d}(x)^{T}}{2}, \quad R_{d}(x)=-\frac{Q_{d}(x)+Q_{d}(x)^{T}}{2}
$$

Furthermore, the damping matrix $R_{d}(x)$ in equation (3) fulfills:

$$
R_{d}(x)=R_{d}(x)^{T} \geq 0
$$

The system (2) is then dissipative in the sense that the time derivative

$$
\frac{d \mathcal{H}_{d}(x)}{d t}=-\left[\frac{\partial \mathcal{H}_{d}(x)}{\partial x}\right]^{T} R_{d}(x)\left[\frac{\partial \mathcal{H}_{d}(x)}{\partial x}\right]
$$

is always negative and the Hamiltonian $\mathcal{H}_{d}(x)$ is bounded from below [3, 4]. Consequently, it plays role of Lyapunov function for stabilization at the desired equilibrium $x_{d}$. The following matching equation ${ }^{2}$ that follows from equations (1) and (2) has to be solved to find $u=\beta(x)$ :

$$
f(x)+g(x) \beta(x)=Q_{d}(x) \frac{\partial \mathcal{H}_{d}(x)}{\partial x}
$$

\footnotetext{
${ }^{2} \mathrm{~A}$ partial differential equation (PDE) [8].
} 
We assume that there exists a full rank left annihilator of $g(x)$ denoted $g(x)^{\perp}$ such that $g(x)^{\perp} g(x)=0$. If $J_{d}(x), R_{d}(x)$ and $\mathcal{H}_{d}(x)$ are chosen such that:

$$
g(x)^{\perp} f(x)=g(x)^{\perp} Q_{d}(x) \frac{\partial \mathcal{H}_{d}(x)}{\partial x}
$$

then the control variable is deduced from the state feedback $\beta(x)$ given by [8]:

$$
\beta(x)=g(x)^{T}\left(g(x) g(x)^{T}\right)^{-1}\left(Q_{d}(x) \frac{\partial \mathcal{H}_{d}(x)}{\partial x}-f(x)\right)
$$

Thus, a general methodology for the PBA in the port Hamiltonian framework is derived from equations (3)(4) and (6)-(8). Three different guidelines can be considered:

(i) We first choose an appropriate Hamiltonian storage function $\mathcal{H}_{d}(x)$. The matrix $Q_{d}(x)$ fulfilling (3)(4) has to be found by considering (7). The feedback $u$ is then synthesized using (8) [14, 41].

(ii) We choose an appropriate matrix $Q_{d}(x)$ fulfilling $(3)(4)$. The Hamiltonian storage function $\mathcal{H}_{d}(x)$ remains to be found by considering (7). From this the feedback $u$ is obtained using (8) [21].

(iii) The matrix $Q_{d}(x)$ fulfilling (3)(4) and the Hamiltonian storage function $\mathcal{H}_{d}(x)$ are simultaneously solved by considering (7). The feedback $u$ is then given by (8) [31]. This guideline becomes quite difficult to implement as degrees of freedom increase [8].

In what follows, we shall show that the PBA is useful, not only for controller synthesis but also asymptotic observers design of a class of the non isothermal CSTR with chemical reactions. The use of the PBA with the guideline (i) is applied.

\section{The CSTR case study with chemical transformation}

\subsection{The CSTR modelling}

Consider a CSTR with $n_{r}$ chemical reactions ${ }^{3}$ with $n_{c}$ active components $\mathcal{C}_{i}$ of molar mass $M_{i}\left(i=1,2, \ldots, n_{c}\right)$. Such a reaction network is character-

\footnotetext{
${ }^{3}$ Without loss of generality, we assume that all considered reactions are irreversible.
} 
ized by the following reaction invariant :

$$
\sum_{j=1}^{n_{c}} \nu_{i j} M_{i}=0, i=1,2, \ldots, n_{r}
$$

where $\nu_{i j}$ is the signed stoichiometric coefficient of species $j$ as it enters in reaction $i[22,21]$. For modelling purposes, we make the following hypotheses :

(H1) The fluid mixture is isobaric, ideal and incompressible.

(H2) The heat flow from the jacket to the reactor is given by :

$$
\dot{Q}_{J}=\lambda\left(T_{J}-T\right)
$$

where $\lambda>0$ is the heat exchange coefficient. The jacket temperature $T_{J}$ is the only control variable.

(H3) The reactor is fed by the species $k(k=1,2, \ldots)$ at a fixed temperature $T_{I}$ and dilution rate $d$. The specific heat capacities $c_{p k}(k=1,2, \ldots)$ are assumed to be constant.

Remark 1. Any reversible reaction $l\left(l \in\left\{1, \ldots, n_{r}\right\}\right)$ of the network (9) can be considered to be irreversible [38] when we define the reduced reaction rate:

$$
r_{l}=\left(r_{l}\right)^{(f)}-\left(r_{l}\right)^{(r)}
$$

where $\left(r_{l}\right)^{(f)}$ and $\left(r_{l}\right)^{(r)}$ are the forward and reserve reaction rates respectively.

Under (H1), the energy balance is written using the enthalpy $H$. Hence the material and energy balances are finally given as follows $[23,12,15,17]$ :

$$
\left\{\begin{aligned}
\frac{d N_{1}}{d t} & =d\left(N_{1 I}-N_{1}\right)+\sum_{j=1}^{n_{r}} \nu_{1 j} r_{j} \\
\frac{d N_{2}}{d t} & =d\left(N_{2 I}-N_{2}\right)+\sum_{j=1}^{n_{r}} \nu_{2 j} r_{j} \\
\vdots & \\
\frac{d N_{n}}{d t} & =d\left(N_{n_{c} I}-N_{n_{c}}\right)+\sum_{j=1}^{n_{r}} \nu_{n j} r_{j} \\
\frac{d H}{d t} & =d\left(H_{I}-H\right)+\dot{Q}_{J}
\end{aligned}\right.
$$


where:

- $N_{i}$ is mole number of species $i\left(i=1, \ldots, n_{c}\right)$;

- $H$ and $r_{j}$ represent the total enthalpy and the reaction rate of the reaction $j\left(j=1, \ldots, n_{r}\right)$;

- $d$ stands for the dilution rate which is assumed to be constant. The subscript $I$ written in equation (12) denotes "Inlet".

Remark 2. Species $\boldsymbol{N}_{\text {ot }}$ that are Inert and/or Catalyst can be added to the dynamics by setting:

$$
\frac{d \boldsymbol{N}_{o t}}{d t}=d\left(\boldsymbol{N}_{o t I}-\boldsymbol{N}_{o t}\right)
$$

where $\boldsymbol{N}_{\text {ot }}$ is a vector containing all these species. But we can easily check that the differential equation (13) is stable and the states converge to 0 . We shall therefore only consider the dynamics of $n_{c}$ active species (12) from the point of view of chemical reaction. However, the presence of Inert and/or Catalyst should be considered in the energy balance since the total enthalpy $H$ in definition (14), the total heat capacity $C_{p}$ in definition (17) and the total mass $m_{t}$ depend not only on $\left(N_{1}, \ldots, N_{n_{c}}\right)$ but also $\boldsymbol{N}_{\text {ot }}$.

Remark 3. The total enthalpy $H$ of the reaction system is given by:

$$
H=\sum_{i} h_{i}(T) N_{i}
$$

with $h_{i}(T)=c_{p i}\left(T-T_{\text {ref }}\right)+h_{\text {iref }}$ where $T_{\text {ref }}$ and $h_{\text {iref }}$ are the reference values. By using the local equilibrium hypothesis, the energy balance $\frac{d H}{d t}$ in (12) can be rewritten in terms of temperature [15] so that:

$$
\frac{d T}{d t}=\frac{\sum_{j=1}^{r}\left(-\Delta H_{R j}\right) r_{j}}{C_{p}}+d\left(T_{I}-T\right) \frac{C_{p I}}{C_{p}}+\frac{1}{C_{p}} \dot{Q}_{J}
$$

where

$$
\Delta H_{R j}=\sum_{i=1}^{n_{c}} \nu_{i j} h_{i}(T)
$$


represents the enthalpy of the chemical reaction $j\left(j=1, \ldots, n_{r}\right)$ and,

$$
C_{p}=\sum_{i} c_{p i} N_{i}
$$

is the total heat capacity.

The system dynamics with state variables $\left(H, N_{1}, \ldots, N_{n_{c}}\right)$ given by equation (12) or $\left(T, N_{1}, \ldots, N_{n_{c}}\right)$ defined by equations (15) and (12) are mathematically equivalent due to definition (14). The dynamical representation corresponding to the state vector $\left(T, N_{1}, \ldots, N_{n_{c}}\right)$ given by ODEs $(12)$ and (15) will be used for controller synthesis. Asymptotic observer design will be solved with the dynamics corresponding to the state vector $\left(H, N_{1}, \ldots, N_{n_{c}}\right)$ in equation (12). The transient behaviour of the differential equation (13) is considered for the energy balance in both cases.

Example 1. We consider the production of cyclopentenol $\mathrm{C}_{5} \mathrm{H}_{7} \mathrm{OH}$ from cyclopentadiene $\mathrm{C}_{5} \mathrm{H}_{6}$ by sulfuric acid-catalyzed addition of water in a dilute solution [18]. The total mass of the liquid phase mixture $m_{t}$ is assumed to be constant. The process is described by the Van de Vusse reaction system [39]. The stoichiometry is written as in (9) with $n_{r}=3$ and $n_{c}=5$ :

$$
\begin{aligned}
& \underbrace{\mathrm{C}_{5} \mathrm{H}_{6}}_{M_{1}}+\underbrace{\mathrm{H}_{2} \mathrm{O}}_{M_{5}} \stackrel{H^{+}}{\rightarrow} \underbrace{\mathrm{C}_{5} \mathrm{H}_{7} \mathrm{OH}}_{M_{2}}+\underbrace{\mathrm{H}_{2} \mathrm{O}}_{M_{5}} \stackrel{H^{+}}{\rightarrow} \underbrace{\mathrm{C}_{5} \mathrm{H}_{8}(\mathrm{OH})_{2}}_{M_{3}} \\
& 2 \underbrace{\mathrm{C}_{5} \mathrm{H}_{6}}_{M_{1}} \rightarrow \underbrace{\mathrm{C}_{10} \mathrm{H}_{12}}_{M_{4}}
\end{aligned}
$$

The system dynamics (12) with 5 active species is given by:

$$
\left\{\begin{aligned}
\frac{d N_{1}}{d t} & =d\left(N_{1 I}-N_{1}\right)-r_{1}-2 r_{3} \\
\frac{d N_{2}}{d t} & =d\left(N_{2 I}-N_{2}\right)+r_{1}-r_{2} \\
\frac{d N_{3}}{d t} & =d\left(N_{3 I}-N_{3}\right)+r_{2} \\
\frac{d N_{4}}{d t} & =d\left(N_{4 I}-N_{4}\right)+r_{3} \\
\frac{d N_{5}}{d t} & =d\left(N_{5 I}-N_{5}\right)-r_{1}-r_{2} \\
\frac{d H}{d t} & =d\left(H_{I}-H\right)+\dot{Q}_{J}
\end{aligned}\right.
$$

Note that sulfuric acid is present as a catalyst. From Remark 2, we therefore have:

$$
\frac{d N_{o t}}{d t}=d\left(N_{o t I}-N_{o t}\right)
$$


In differential equations (19) and (20), we have $d=\frac{q_{m}}{m_{t}}$ and $N_{i I}=x_{i I} \frac{m_{t}}{M_{i}}$ where $\sum_{i} x_{i I}=1$ and $q_{m}$ is the mass flow rate. Finally, the energy balance $\frac{d H}{d t}$ in equation (19) is written in terms of the temperature $T$ (see Remark 3) so that:

$$
\frac{d T}{d t}=\frac{\sum_{j=1}^{3}\left(-\Delta H_{R j}\right) r_{j}}{C_{p}}+d\left(T_{I}-T\right) \frac{C_{p I}}{C_{p}}+\frac{1}{C_{p}} \dot{Q}_{J}
$$

where:

$$
\left\{\begin{array}{l}
\Delta H_{R 1}=-h_{1}-h_{5}+h_{2}>0 \\
\Delta H_{R 2}=-h_{2}-h_{5}+h_{3}<0 \\
\Delta H_{R 3}=-2 h_{1}+h_{4}<0
\end{array}\right.
$$

and,

$$
C_{p}=c_{p 1} N_{1}+c_{p 2} N_{2}+c_{p 3} N_{3}+c_{p 4} N_{4}+c_{p 5} N_{5}+c_{p o t} N_{o t}
$$

\subsection{Preliminaries}

The following assumptions are now made to characterise the dynamical behaviour of the system (12):

(A1) The reaction rates $r_{j}\left(j=1, \ldots, n_{r}\right)$ are described by the mass action laws,

$$
r_{j}=k_{j}(T) F_{j}\left(N_{1}^{\left|\nu_{1 j}\right|}, N_{2}^{\left|\nu_{2 j}\right|}, \ldots\right), j=1, \ldots, n_{r}
$$

where $F_{j}\left(j=1, \ldots, n_{r}\right)$ are nonlinear functions with respect to their arguments and $k_{j}(T)\left(j=1, \ldots, n_{r}\right)$ are reaction rate constants fulfilling the condition that $k_{j}(T)$ is monotone, non-negative and bounded in accordance to thermodynamic principles $[17,12,15]$ so that:

$$
\lim _{T \rightarrow 0} k_{j}(T)=0 \quad \text { and } \lim _{T \rightarrow+\infty} k_{j}(T)=k_{j \max }
$$

The Arrhenius law

$$
k_{j}(T)=k_{0 j} \exp \left(\frac{-k_{1 j}}{T}\right)
$$

where $k_{0 j}$ is the kinetic constant and $k_{1 j}$ is the activation temperature, is compatible with the limits in equation (25).

(A2) The temperature and mole numbers are non-negative. 
Assumption (A2) describes measurable physical quantities [30] and implies that the CSTR is a positive system. In what follows, we first present the following results which are instrumental in proving the main results of this work.

\subsubsection{Boundedness of material dynamics}

Lemma 1 generalizes the results of Theorem 2.1 (i) presented in [19] by considering multi-component homogeneous mixtures.

Lemma 1. The domain $\Omega=\left\{N_{1}, \ldots, N_{n_{c}} \mid 0 \leq \sum_{i=1}^{n_{c}} M_{i} N_{i} \leq \sum_{i=1}^{n_{c}} M_{i} N_{i I}\right\}$ is positively invariant.

Proof. Define $\eta=\sum_{i=1}^{n_{c}} M_{i} N_{i}$. By using the mass conservation property given by equation (9), we obtain from equation (12):

$$
\frac{d \eta}{d t}=d\left(\sum_{i=1}^{n_{c}} M_{i} N_{i I}-\eta\right)
$$

$\eta(t) \leq \sum_{i=1}^{n_{c}} M_{i} N_{i I}$ for all $\eta(t=0) \leq \sum_{i=1}^{n_{c}} M_{i} N_{i I}$ since $d>0$. Using (A2), one gets $\eta(t) \geq 0$. The latter completes the proof.

\subsubsection{Stability of the isothermal dynamics}

Let $\left(N_{1 d}, N_{2 d}, \ldots, N_{N d}, T_{d}\right)$ be the steady state of the reaction system defined by equations (12) and (15). Let us note that possible steady states are calculated by considering that all time derivatives vanish and that there may be more than one stationary solution to the problem [12]. An additional assumption (used in [19, 29, 30] or recently [12]) is considered:

(A3) For the isothermal dynamics $\left(T=T_{d}\right)$, the system dynamics (12) admits a single equilibrium point $\left(N_{1 d}, \ldots, N_{n d}\right)$ which is globally asymptotically stable.

From a control point of view, we can show by means of Lyapunov converse theorems [1] together with the above assumption, that there exists a positive function $\mathcal{V}\left(N_{1}, \ldots, N_{n_{c}}\right)$ with $\frac{d \mathcal{V}}{d t}<0$ along the isothermal dynamics. Several industrial chemical reaction processes verify this assumption. Let us illustrate with the Van de Vusse reaction system in Example 1. 
Example 2. We rewrite the isothermal dynamics derived from equation (19) into the explicit form using (A1) so that:

$$
\left\{\begin{aligned}
\frac{d N_{1}}{d t} & =d\left(N_{1 I}-N_{1}\right)-k_{1}\left(T_{d}\right) N_{1}-2 k_{3}\left(T_{d}\right) N_{1}^{2} \\
\frac{d N_{2}}{d t} & =d\left(N_{2 I}-N_{2}\right)+k_{1}\left(T_{d}\right) N_{1}-k_{2}\left(T_{d}\right) N_{2} \\
\frac{d N_{3}}{d t} & =d\left(N_{3 I}-N_{3}\right)+k_{2}\left(T_{d}\right) N_{2} \\
\frac{d N_{4}}{d t} & =d\left(N_{4 I}-N_{4}\right)+k_{3}\left(T_{d}\right) N_{1}^{2} \\
\frac{d N_{5}}{d t} & =d\left(N_{5 I}-N_{5}\right)-k_{1}\left(T_{d}\right) N_{1}-k_{2}\left(T_{d}\right) N_{2}
\end{aligned}\right.
$$

The existence of the positive-definite function $\mathcal{V}\left(N_{1}, \ldots, N_{n_{c}}\right)$ is derived by considering the separable dynamics of (27). Indeed the dynamics on $N_{1}(27)$ can be rewritten as follows:

$$
\frac{d N_{1}}{d t}=-2 k_{3}\left(T_{d}\right)\left(N_{1}-N_{1 d}\right)\left(N_{1}-\bar{N}_{1 d}\right)
$$

where $N_{1 d}>0$ and $\bar{N}_{1 d}<0$ are roots of the second-order polynomial equation that follow by setting $\frac{d N_{1}}{d t}=0$ in equation (27):

$$
\left\{\begin{array}{l}
N_{1 d}=\frac{\left(d+k_{1}\left(T_{d}\right)\right)-\sqrt{\left(d+k_{1}\left(T_{d}\right)\right)^{2}+8 d k_{3}\left(T_{d}\right) N_{1 I}}}{-4 k_{3}\left(T_{d}\right)} \\
\bar{N}_{1 d}=\frac{\left(d+k_{1}\left(T_{d}\right)\right)+\sqrt{\left(d+k_{1}\left(T_{d}\right)\right)^{2}+8 d k_{3}\left(T_{d}\right) N_{1 I}}}{-4 k_{3}\left(T_{d}\right)}
\end{array}\right.
$$

Lemma 1 shows that there exits a positive constant $\varrho>0$ so that (28) can be rewritten as follows:

$$
\frac{d N_{1}}{d t} \leq-2 \varrho k_{3}\left(T_{d}\right)\left(N_{1}-N_{1 d}\right)
$$

It is now clear that the positive-definite function $\mathcal{V}_{1}\left(N_{1}\right)=\frac{1}{2}\left(N_{1}-N_{1 d}\right)^{2}$ is a Lyapunov function candidate for the stabilization of (30) at $N_{1 d}$. The same argument sequentially applies to $N_{2}, N_{3}, N_{4}$ and $N_{5}$. Finally, the (global) Lyapunov function of the isothermal dynamics (27) is defined so that:

$$
\mathcal{V}\left(N_{1}, \ldots, N_{n_{c}}\right)=\sum_{k=1}^{5} \mathcal{V}_{k}\left(N_{k}\right)
$$

In the following we focus our attention on nonlinear control and state estimation problems of non isothermal CSTR (27). These two problems will be effectively solved in the framework of the passivity theory. 


\section{Main results}

\subsection{Controller design}

For controller synthesis, it is convenient to let the state vector $x=$ $\left(N_{1}, \ldots, N_{n_{c}}, T\right)$ represent the reaction system dynamics (15)(12). The dynamics (1) is then obtained with:

$$
f(x)=\left(\begin{array}{c}
d\left(N_{1 I}-N_{1}\right)+\sum_{j=1}^{n_{r}} \nu_{1 j} r_{j} \\
d\left(N_{2 I}-N_{2}\right)+\sum_{j=1}^{n_{r}} \nu_{2 j} r_{j} \\
\vdots \\
d\left(N_{n_{c} I}-N_{n_{c}}\right)+\sum_{j=1}^{n_{r}} \nu_{n j} r_{j} V \\
\frac{\sum_{j=1}^{n_{r}}\left(-\Delta H_{R j}\right) r_{j}-\lambda T}{C_{p}}+d\left(T_{I}-T\right) \frac{C_{p I}}{C_{p}}
\end{array}\right), \quad g(x)=\left(\begin{array}{c}
0 \\
0 \\
\vdots \\
0 \\
\frac{\lambda}{C_{p}}
\end{array}\right) \quad \text { and } u=T_{J}
$$

The PBA with the guideline (i) (in Section 2) will be used to design a passive nonlinear controller for the stabilization of the reaction system (1) with (32) at a given desired state $x_{d}$. The problem first consists of choosing an appropriate closed loop Hamiltonian storage function $\mathcal{H}_{d}(x)$. Let us note that in previous works $[19,29]$, a Lyapunov function candidate based on thermal deviation $\frac{1}{2}\left(T-T_{d}\right)^{2}$ is considered for the temperature stabilization problem. Farschman and coworkers in [32] have proposed an inventory-based quadratic storage function $\frac{1}{2}\left(x-x_{d}\right)^{2}$ for control of chemical process systems. In $[21,15]$, the thermodynamic availability and its individual contributions have been used as the desired closed loop storage functions. We now show that the matrix $Q_{d}(x)$ can be found using the PBA with the Hamiltonian function:

$$
\mathcal{H}_{d}(x)=\mathcal{H}_{d}\left(T, N_{1}, \ldots, N_{n_{c}}\right)=\frac{\left(T-T_{d}\right)^{2}}{2}\left(1+\sum_{i=1}^{n_{c}} K_{i}\left(N_{i}-N_{i d}\right)^{2}\right)
$$

where $K_{i} \geq 0$. One consequence of definition (33) is that sufficient damping is introduced to allow the stabilization problem to be accomplished with 
a smooth control law in terms of the amplitude and variation rate. The proposed controller therefore generalizes the one obtained from [19] which uses input constraints and non-smooth controls. It also allows us to rewrite the closed loop dynamics (1) with (32) in a port Hamiltonian representation as seen in Proposition 1 below.

Proposition 1. The reaction system described by equations (1) and (32) is exponentially stabilized at the desired state $x_{d}=\left(N_{1 d}, \ldots, N_{n d}, T_{d}\right)$ with the following state feedback control law:

$$
\begin{aligned}
T_{J}=T+ & \frac{1}{\lambda}\left\{C_{p}\left[-\left(\frac{\partial \mathcal{H}_{d}}{\partial T}\right)^{-1} \sum_{i=1}^{n_{c}} \frac{\partial \mathcal{H}_{d}}{\partial N_{i}} \frac{d N_{i}}{d t}-K_{T} \frac{\partial \mathcal{H}_{d}}{\partial T}\right]\right. \\
& \left.-\left(\sum_{j=1}^{n_{r}}\left(-\Delta H_{R j}\right) r_{j}+d\left(T_{I}-T\right) C_{p I}\right)\right\}
\end{aligned}
$$

where $K_{T}>0$ is a tuning parameter. Furthermore, the closed loop dynamics are represented in the passive Hamiltonian format so that:

$$
\frac{d x}{d t}=\left[J_{d}(x)-R_{d}(x)\right] \frac{\partial \mathcal{H}_{d}(x)}{\partial x}
$$

where:

$$
\begin{aligned}
& J_{d}(x)=\left(\begin{array}{ccccc}
0 & \ldots & & 0 & \left(\frac{\partial \mathcal{H}_{d}}{\partial T}\right)^{-1} \frac{d N_{1}}{d t} \\
\vdots & \ddots & & \vdots & \vdots \\
0 & \ldots & & 0 & \left(\frac{\partial \mathcal{H}_{d}}{\partial T}\right)^{-1} \frac{d N_{n_{c}}}{d t} \\
-\left(\frac{\partial \mathcal{H}_{d}}{\partial T}\right)^{-1} \frac{d N_{1}}{d t} & \ldots & -\left(\frac{\partial \mathcal{H}_{d}}{\partial T}\right)^{-1} \frac{d N_{n_{c}}}{d t} & 0
\end{array}\right) \\
& R_{d}(x)=\left(\begin{array}{cccc}
0 & \ldots & 0 & 0 \\
\vdots & \ddots & \vdots & \vdots \\
0 & \ldots & 0 & 0 \\
0 & \ldots & 0 & K_{T}
\end{array}\right)
\end{aligned}
$$

and $\mathcal{H}_{d}(x)$ is given by (33).

Proof. By using the PBA as described in Section 2, we have $Q_{d}(x)=$ $\left(q_{i j}(x)\right)_{i, j=1 \ldots\left(n_{c}+1\right)}$ and $g^{\perp}(x)=\operatorname{diag}(1, \ldots, 1,0) \in \mathbb{R}^{\left(n_{c}+1\right) \times\left(n_{c}+1\right)}$. 
The matching equations from (7) give the following partial differential equations :

$$
\left\{\begin{array}{c}
q_{11}(x) \frac{\partial \mathcal{H}_{d}}{\partial N_{1}}+\ldots+q_{1 n_{c}}(x) \frac{\partial \mathcal{H}_{d}}{\partial N_{n_{c}}}+q_{1\left(n_{c}+1\right)}(x) \frac{\partial \mathcal{H}_{d}}{\partial T}=\frac{d N_{1}}{d t} \\
\vdots \\
q_{n_{c} 1}(x) \frac{\partial \mathcal{H}_{d}}{\partial N_{1}}+\ldots+q_{n n}(x) \frac{\partial \mathcal{H}_{d}}{\partial N_{n_{c}}}+q_{n_{c}\left(n_{c}+1\right)}(x) \frac{\partial \mathcal{H}_{d}}{\partial T}=\frac{d N_{n_{c}}}{d t}
\end{array}\right.
$$

The number of equations equals $n_{c}$ with $n_{c} \times\left(n_{c}+1\right)$ unknown variables $q_{i j}(x)$. Hence this system has an infinite number of solutions. A simple solution is found using the negative definiteness of the matrix $Q_{d}(x)$ as follows:

(a) set $q_{i j}(x)=q_{j i}(x)=0$ for $i, j=1 \ldots n_{c}$;

(b) then set $q_{i\left(n_{c}+1\right)}(x)=-q_{\left(n_{c}+1\right) i}(x)=\left(\frac{\partial \mathcal{H}_{d}}{\partial T}\right)^{-1} \frac{d N_{i}}{d t}$ for $i=1 \ldots n_{c}$;

(c) and finally choose $q_{\left(n_{c}+1\right)\left(n_{c}+1\right)}(x)=-K_{T}$.

It follows that $q_{i\left(n_{c}+1\right)}(x), i=1 \ldots n_{c}$ is well defined in the limit $\frac{\partial \mathcal{H}_{d}}{\partial T} \rightarrow 0$ (refer to equation (41) below). The structure matrices $J_{d}(x)(36)$ and $R_{d}(x)$ (37) are computed by using (3). Finally, the feedback law is derived from (8) with $J_{d}(x)$ defined in equation $(36), R_{d}(x)$ defined in equation (37) and $\mathcal{H}_{d}(x)$ given in equation (33):

$$
\begin{aligned}
& C_{p}\left[-\left(\frac{\partial \mathcal{H}_{d}}{\partial T}\right)^{-1} \frac{\partial \mathcal{H}_{d}}{\partial N_{1}} \frac{d N_{1}}{d t}-\ldots-\left(\frac{\partial \mathcal{H}_{d}}{\partial T}\right)^{-1} \frac{\partial \mathcal{H}_{d}}{\partial N_{n_{c}}} \frac{d N_{n_{c}}}{d t}\right. \\
& \left.-K_{T} \frac{\partial \mathcal{H}_{d}}{\partial T}\right]-\left(\sum_{j=1}^{n_{r}}\left(-\Delta H_{R j}\right) r_{j}+d\left(T_{I}-T\right) C_{p I}\right)=\dot{Q}_{J}
\end{aligned}
$$

Using (H2) with $u=T_{J}$ leads to the feedback law (34). Let us note that the feedback law (34) is well-defined when $\mathcal{H}_{d}(x)$ is defined by equation (33). The function $\mathcal{H}_{d}(x)$ is positive definite and its time derivative satisfies

$$
\frac{d \mathcal{H}_{d}(x)}{d t}=-K_{T}\left(\frac{\partial \mathcal{H}_{d}}{\partial T}\right)^{2}<0, \forall T \neq T_{d}
$$

From Lemma 1 and equation (33) it follows that there exists $\varsigma>0$ so that:

$$
\frac{d \mathcal{H}_{d}(x)}{d t} \leq-\varsigma \mathcal{H}_{d}(x)
$$


The stability proof immediately follows invoking La Salle's invariance principle [1] and (A3). We now develop some important limiting properties of the closed loop system to show that the control law proposed in equation (34) is well-defined. First, it follows from the development above that the closed loop dynamics of the temperature $T$ with the feedback law given in equation (34) can be rewritten as follows:

$$
\frac{d T}{d t}=-\left(\frac{\partial \mathcal{H}_{d}}{\partial T}\right)^{-1} \frac{d N_{1}}{d t} \frac{\partial \mathcal{H}_{d}}{\partial N_{1}}-\ldots-\left(\frac{\partial \mathcal{H}_{d}}{\partial T}\right)^{-1} \frac{d N_{n_{c}}}{d t} \frac{\partial \mathcal{H}_{d}}{\partial N_{n_{c}}}-K_{T} \frac{\partial \mathcal{H}_{d}}{\partial T}
$$

Second, we note that it follows from the definition of $\mathcal{H}_{d}(x)$ in equation (33) that:

$$
\frac{\partial \mathcal{H}_{d}}{\partial T} \rightarrow 0 \Leftrightarrow T \rightarrow T_{d}
$$

and,

$$
\lim _{T \rightarrow T_{d}} \frac{\partial \mathcal{H}_{d}}{\partial N_{i}} \rightarrow 0, i=1, \ldots, n_{c}
$$

From (33) we also have:

$$
\mathcal{H}_{d}\left(T=T_{d}, N_{1}, \ldots, N_{n_{c}}\right)=0
$$

and

$$
\left.\frac{d \mathcal{H}_{d}}{d t}\right|_{\left(T=T_{d}, N_{1}, \ldots, N_{n_{c}}\right)}=0
$$

We have shown that $\mathcal{H}_{d}(x)$ is a Lyapunov function with (38) for the stabilization of the reactor temperature $T$. As a consequence, we obtain $\lim _{T \rightarrow T_{d}} \frac{d T}{d t}=$ $\lim _{\frac{\partial \mathcal{H}_{d}}{\partial T} \rightarrow 0} \frac{d T}{d t}=0$ and thus we deduce from $(40)$ :

$$
\left\{\begin{array}{l}
\lim _{\frac{\partial \mathcal{H}_{d}}{\partial T} \rightarrow 0}\left(\frac{\partial \mathcal{H}_{d}}{\partial T}\right)^{-1} \frac{d N_{1}}{d t}<\infty \\
\vdots \\
\lim _{\frac{\partial \mathcal{H}_{d}}{\partial T} \rightarrow 0}\left(\frac{\partial \mathcal{H}_{d}}{\partial T}\right)^{-1} \frac{d N_{n_{c}}}{d t}<\infty
\end{array}\right.
$$

The latter completes the proof. 
Remark 4. The feedback law (34) becomes similar to the one proposed by Viel and coworkers [19] when the constraints are ignored and $K_{1}=\ldots=$ $K_{n_{c}}=0$ in $\mathcal{H}_{d}(x)$ as defined by equation (33). In this case, the stabilization is dominated by the regulation of the thermal part in accordance with the Assumption (A3). In the general case $\left(\prod_{i=1}^{n_{c}} K_{i} \neq 0\right)$, we may use the gains to shape the amplitude and variation rate of the control input through the presence of the material balances given by equations (33) and (34).

\subsection{Asymptotic observers}

We now consider a situation where only the reactor temperature $T$ and a subset of the concentrations are measured. In this case we have to design an observer to reconstruct other missing variables within the mixture. We also need to find a method to determine how many and which concentrations need to be measured. The main feature of the proposed observer is that it is independent of the system kinetics and is called asymptotic observers. These asymptotic observers were first proposed in [37] for simplified CSTR models and developed further in [27] for more general CSTR models. However, feedback law was not considered in these contributions and there is a question whether the use of the estimated states in feedback gives stable control. In what follows we show, analytically and/or simulations, that the estimated state variables exponentially converge to their exact values with and without feedback. Let us reconsider the original system (12) and rewrite it into the following form:

$$
(\Sigma)\left\{\begin{aligned}
\frac{d H}{d t} & =d\left(H_{I}-H\right)+\dot{Q}_{J} \\
\frac{d \boldsymbol{N}}{d t} & =d\left(\boldsymbol{N}_{I}-\boldsymbol{N}\right)+\boldsymbol{\nu} \boldsymbol{r}
\end{aligned}\right.
$$

where $\boldsymbol{N}=\left(N_{1}, \ldots, N_{n_{c}}\right)^{T}$ is the vector of mole numbers. $\boldsymbol{\nu}=\left(\nu_{i j}\right)_{\substack{i=1 \ldots n_{c} \\ j=1 \ldots n_{r}}}$ is the matrix of stoichiometric coefficients and $\boldsymbol{r}=\left(r_{1}, \ldots, r_{n_{r}}\right)^{T}$ is the vector composed of chemical reaction rates. The following additional assumption is made [37, 27]:

(A4) The reaction network (9) with $n_{r}<n_{c}$ is independent so that,

$$
\operatorname{rank}(\boldsymbol{\nu})=n_{r}
$$


And $\left(n_{r}-1\right)$ concentrations and the reactor temperature $T$ are assumed to be available for the online measurement ${ }^{4}$.

We have the following lemma.

Lemma 2. There exists an $n_{c} \times n_{c}$ matrix $\Theta$ so that:

$$
\Theta=\left(\frac{\boldsymbol{v}_{n_{r} \times n_{c}}}{\boldsymbol{\nu}_{\left(n_{c}-n_{r}\right) \times n_{c}}}\right)_{n_{c} \times n_{c}}
$$

where the following equalities hold:

$$
\boldsymbol{v} \boldsymbol{\nu}=\mathbb{I}_{n_{r} \times n_{r}}
$$

and

$$
\boldsymbol{\nu}^{\perp} \boldsymbol{\nu}=\mathbf{0}_{\left(n_{c}-n_{r}\right) \times n_{r}}
$$

where $\mathbb{I}_{n_{r} \times n_{r}}$ and $\mathbf{0}_{\left(n_{c}-n_{r}\right) \times n_{r}}$ are the identity and the zero matrices respectively.

Proof. The proof immediately follows using Assumption A4. Indeed it can be shown that the matrix $\Theta$ is directly derived by Gauss elimination.

Example 3. Let us consider the Van de Vusse reaction system given Example 1. Its dynamics (19) can be re-expressed as (42), where:

$$
\boldsymbol{\nu}=\left(\begin{array}{rrr}
-1 & 0 & -2 \\
1 & -1 & 0 \\
0 & 1 & 0 \\
0 & 0 & 1 \\
-1 & -1 & 0
\end{array}\right) \quad \text { and } \boldsymbol{r}=\left(r_{1}, r_{2}, r_{3}\right)^{T}
$$

After some manipulation we have:

$$
\Theta=\left(\begin{array}{rrrrr}
0 & 1 & 1 & 0 & 0 \\
0 & 0 & 1 & 0 & 0 \\
-\frac{1}{2} & -\frac{1}{2} & -\frac{1}{2} & 0 & 0 \\
\hline \frac{1}{2} & \frac{1}{2} & \frac{1}{2} & 1 & 0 \\
0 & 1 & 2 & 0 & 1
\end{array}\right)=\left(\frac{\boldsymbol{v}_{3 \times 5}}{\boldsymbol{\nu}_{2 \times 5}^{\perp}}\right)_{5 \times 5}
$$

and $\Theta$ verifies equations (44) and (45).

\footnotetext{
${ }^{4}$ That means that $n_{r}$ values are assumed to be measured.
} 
As a consequence of Lemma 2, We state Proposition 2.

Proposition 2. The map from $\mathbb{R}^{n_{c}}$ to $\mathbb{R}^{n_{c}-n_{r}}, \boldsymbol{Z}=\boldsymbol{\nu}^{\perp} \boldsymbol{N}$, reduces the $d y$ namics for $\boldsymbol{N}$ defined by equation (42) to:

$$
\frac{d \boldsymbol{Z}}{d t}=d\left(\boldsymbol{Z}_{I}-\boldsymbol{Z}\right)
$$

where $\boldsymbol{Z}_{I}=\boldsymbol{\nu}^{\perp} \boldsymbol{N}_{I}$ and $\boldsymbol{\nu}^{\perp}$ are given in equation (43). Furthermore, the reduced dynamics are independent of the chemical reaction kinetics.

Proof. The proof immediately follows by multiplying equation (42) with $\boldsymbol{\nu}^{\perp}$ defined by equation (43) (see also [37, 27]).

In the remaining of the paper, we let $\mathbb{N}=\left\{1, \ldots, n_{c}\right\}$ be the set of indices for chemical species of the mixture described by the invariant (9) and the differential equations (42). It is worth noting that there exists a disjoint partitioning $\mathcal{I}, \mathcal{J} \subset \mathbb{N}$ with $\left(n_{r}-1\right)$ and $\left(n_{c}-n_{r}+1\right)$ elements respectively so that:

$$
\begin{aligned}
& \mathcal{I} \cap \mathcal{J}=\emptyset \\
& \mathcal{I} \cup \mathcal{J}=\mathbb{N}
\end{aligned}
$$

where $\mathcal{I}$ and $\mathcal{J}$ refer to the subsets of $\left(n_{r}-1\right)$ measured mole numbers ${ }^{5}$ and $\left(n_{c}-n_{r}+1\right)$ remaining mole numbers to be estimated respectively. As a consequence, we can write from definition (14) and Proposition 2:

$$
\left\{\begin{array}{c}
H=\boldsymbol{h}_{\mathcal{I}}^{T} \boldsymbol{N}_{\mathcal{I}}+\boldsymbol{h}_{\mathcal{J}}^{T} \boldsymbol{N}_{\mathcal{J}}+\boldsymbol{h}_{o t}^{T} \boldsymbol{N}_{o t} \\
\boldsymbol{Z}=\left(\boldsymbol{\nu}^{\perp}\right)_{\mathcal{I}} \boldsymbol{N}_{\mathcal{I}}+\left(\boldsymbol{\nu}^{\perp}\right)_{\mathcal{J}} \boldsymbol{N}_{\mathcal{J}}
\end{array}\right.
$$

where $\left(\boldsymbol{\nu}^{\perp}\right)_{\mathcal{I}}$ and $\left(\boldsymbol{\nu}^{\perp}\right)_{\mathcal{J}}$ are submatrices of the matrix $\boldsymbol{\nu}^{\perp}$ formed by selecting columns corresponding to the marked mole numbers. The convergence of the estimates $\hat{N}_{j}, \forall j \in \mathcal{J}$ to their exact values is shown in Proposition 3.

Proposition 3. If the square matrix defined by

$$
\mathcal{O}=\left(\begin{array}{c}
\boldsymbol{h}_{\mathcal{J}}^{T} \\
\left(\boldsymbol{\nu}^{\perp}\right)_{\mathcal{J}}
\end{array}\right)_{\left(n_{c}-n_{r}+1\right) \times\left(n_{c}-n_{r}+1\right)}
$$

\footnotetext{
${ }^{5}$ It does not include the temperature.
} 
fulfills the following condition,

$$
\operatorname{rank}(\mathcal{O})=n_{c}-n_{r}+1
$$

then the states of the system $(\Sigma)$ defined by equation (42) are asymptotically reconstructed with the asymptotic observer $(\hat{\Sigma})$ :

$$
(\hat{\Sigma})\left\{\begin{array}{l}
\frac{d \hat{H}}{d t}=d\left(H_{I}-\hat{H}\right)+\dot{Q}_{J} \\
\frac{d \hat{\boldsymbol{Z}}}{d t}=d\left(\boldsymbol{Z}_{I}-\hat{\boldsymbol{Z}}\right)
\end{array}\right.
$$

The convergence rate of each $\hat{N}_{j}, \forall j \in \mathcal{J}$ defined from $(\hat{\Sigma})$ to the exact value is exponential with the time constant $\tau=\frac{1}{2 d}$. Furthermore, the results above hold whether the system is operated in open or closed loop.

Proof. Let us define $\boldsymbol{\epsilon}(t)=\left(\begin{array}{c}\epsilon_{\tilde{H}} \\ \epsilon_{\tilde{\boldsymbol{Z}}}\end{array}\right)=\left(\begin{array}{c}\hat{H}-H \\ \hat{\boldsymbol{Z}}-\boldsymbol{Z}\end{array}\right) \in \mathbb{R}^{\left(n_{c}-n_{r}+1\right)}$. By subtracting (51) to (42), we get:

$$
\frac{d \boldsymbol{\epsilon}}{d t}=-d \mathbb{I}_{\left(n_{c}-n_{r}+1\right) \times\left(n_{c}-n_{r}+1\right)}\left(\begin{array}{c}
\epsilon_{\tilde{H}} \\
\epsilon_{\tilde{Z}}
\end{array}\right)
$$

with $d>0$. The dynamics of $\boldsymbol{\epsilon}$ is then presented in the port Hamiltonian format (2) where $J(\boldsymbol{\epsilon})=0, R(\boldsymbol{\epsilon})=d \mathbb{I}_{\left(n_{c}-n_{r}+1\right) \times\left(n_{c}-n_{r}+1\right)}$ and the Hamiltonian storage function $\mathcal{H}(\boldsymbol{\epsilon})=\frac{1}{2} \boldsymbol{\epsilon}^{T} \boldsymbol{\epsilon} \geq 0 . \mathcal{H}(\boldsymbol{\epsilon})$ plays a role of a Lyapunov function for the stability of the zero dynamics of $\epsilon$ because:

$$
\frac{d \mathcal{H}(\boldsymbol{\epsilon})}{d t}=-\left(\frac{\partial \mathcal{H}(\boldsymbol{\epsilon})}{\partial \boldsymbol{\epsilon}}\right)^{T} R(\boldsymbol{\epsilon})\left(\frac{\partial \mathcal{H}(\boldsymbol{\epsilon})}{\partial \boldsymbol{\epsilon}}\right)<0
$$

Furthermore, it can be rewritten as follows:

$$
\frac{d \mathcal{H}(\boldsymbol{\epsilon})}{d t}=-d \boldsymbol{\epsilon}^{T} \boldsymbol{\epsilon}=-2 d \mathcal{H}(\boldsymbol{\epsilon}) \Rightarrow \mathcal{H}(\boldsymbol{\epsilon}(t))=\mathcal{H}(\boldsymbol{\epsilon}(t=0)) \exp { }^{-\frac{t}{2 d}}
$$

$\mathcal{H}(\boldsymbol{\epsilon}(t))$ exponentially converges to 0 with the time constant $\tau=\frac{1}{2 d}$ since $d>0$. As a consequence, we have $\boldsymbol{\epsilon}(t) \rightarrow 0$, e.g. $\hat{H} \rightarrow H$ and $\hat{\boldsymbol{Z}} \rightarrow \boldsymbol{Z}$. Using equation (48) together with equation (13), we obtain:

$$
\mathcal{O}\left(\hat{\mathbf{N}}_{\mathcal{J}}-\boldsymbol{N}_{\mathcal{J}}\right)=0
$$


where the matrix $\mathcal{O}$ is defined by equation (49). With condition (50), we conclude:

$$
\hat{\mathbf{N}}_{\mathcal{J}}=\boldsymbol{N}_{\mathcal{J}}
$$

The estimated values $\hat{\mathbf{N}}_{\mathcal{J}}$ are then calculated from the asymptotic observer $(\hat{\Sigma})$ defined in equation (51) using equations (48) and (13):

$$
\hat{\boldsymbol{N}}_{\mathcal{J}}=\mathcal{O}^{-1}\left(\begin{array}{c}
\hat{H}-\boldsymbol{h}_{\mathcal{I}}^{T} \boldsymbol{N}_{\mathcal{I}}-\boldsymbol{h}_{o t}^{T} \hat{\boldsymbol{N}}_{o t} \\
\hat{\boldsymbol{Z}}-\left(\boldsymbol{\nu}^{\perp}\right)_{\mathcal{I}} \boldsymbol{N}_{\mathcal{I}}
\end{array}\right)
$$

It is important to notice that the convergence does not depend on the feedback strategy. The latter completes the proof.

Remark 5. The estimates $\hat{\mathbf{N}}_{\text {ot }}$ of the states $\boldsymbol{N}_{\text {ot }}$ used in equation (53) are derived by using the differential equation (13) so that:

$$
\frac{d \hat{\mathbf{N}}_{o t}}{d t}=d\left(\boldsymbol{N}_{o t I}-\hat{\mathbf{N}}_{o t}\right)
$$

We note that the observability matrix (49) and the full rank condition (50) can be regarded as feasibility conditions for the asymptotic observer [40]. The condition (50) is fulfilled only if the reactions are independent [37, 27], and more precisely if the states to be estimated in $\mathcal{J}$ have intrinsically been involved in the same reactions. Hence the proposed result generalizes and completes the analysis given in $[37,27]$. Let us illustrate this statement via the following example.

Example 4. Example 3 showed that $\mathbb{N}=\{1,2,3,4,5\}$ and

$$
\boldsymbol{\nu}^{\perp}=\left(\begin{array}{ccccc}
\frac{1}{2} & \frac{1}{2} & \frac{1}{2} & 1 & 0 \\
0 & 1 & 2 & 0 & 1
\end{array}\right)
$$

If we choose $\mathcal{I}=\{1,3\}$ and $\mathcal{J}=\{2,4,5\}$ then we get $\mathcal{O}=\left(\begin{array}{rrr}h_{2} & h_{4} & h_{5} \\ \frac{1}{2} & 1 & 0 \\ 1 & 0 & 1\end{array}\right)$.

We can easily check that $\mathcal{O}$ is not full rank because it is not necessarily true that $\operatorname{det}(\mathcal{O})=h_{2}-\frac{1}{2} h_{4}+\frac{1}{2} h_{5} \neq 0$ for non isothermal reactors. Otherwise, if 
we choose $\mathcal{I}=\{3,4\}, \mathcal{J}=\{1,2,5\}$ then we get $\mathcal{O}=\left(\begin{array}{rrr}h_{1} & h_{2} & h_{5} \\ \frac{1}{2} & \frac{1}{2} & 0 \\ 0 & 1 & 1\end{array}\right)$. The latter is full rank because the species $N_{1}, N_{2}$ and $N_{5}$ are effectively involved in the first chemical reaction of the network (18) and thus $\operatorname{det}(\mathcal{O})=\frac{1}{2}\left(h_{1}-\right.$ $\left.h_{2}+h_{5}\right)=-\frac{1}{2} \Delta H_{R 1}<0$ as seen from equation (22).

\section{Illustrative example}

Let us consider a CSTR with one exothermic reaction involving 2 active chemical species $A$ and $B\left(\right.$ e.g. $n_{c}=2$ and $\left.n_{r}=1\right)$ with the stoichiometry:

$$
\nu_{A} M_{A} \rightarrow \nu_{B} M_{B}
$$

The reactor is fed by species $A, B$ and an inert with a fixed inlet temperature $T_{I}$. The balance equations are (see also (12)):

$$
\left\{\begin{array}{l}
\frac{d N_{A}}{d t}=d\left(N_{A I}-N_{A}\right)+\nu_{A} r \\
\frac{d N_{B}}{d t}=d\left(N_{B I}-N_{B}\right)+\nu_{B} r \\
\frac{d H}{d t}=d\left(H_{I}-H\right)+\dot{Q}_{J}
\end{array}\right.
$$

As previously mentioned, the energy balance in equation (56) can be rewritten in terms of temperature as follows:

$$
C_{p} \frac{d T}{d t}=\left(-\Delta H_{R}\right) r+d\left(T_{I}-T\right) C_{p I}+\dot{Q}_{J}
$$

where $\Delta H_{R}=\left(\nu_{B} h_{B}(T)+\nu_{A} h_{A}(T)\right)<0$ is the heat of reaction and $C_{p}=$ $c_{p A} N_{A}+c_{p B} N_{B}+c_{p \text { Inert }} N_{\text {Inert }}$ is the total heat capacity. Finally the dynamics of inert is given by:

$$
\frac{d N_{\text {Inert }}}{d t}=d\left(N_{\text {InertI }}-N_{\text {Inert }}\right) \equiv 0
$$

The numerical values are given in Table 1 [41]. 
Table 1: Parameters of CSTR

\begin{tabular}{lll}
\hline & Numerical value & \\
$c_{p A}$ & $221.9\left(\mathrm{JK}^{-1} \mathrm{~mol}^{-1}\right)$ & Heat capacity of species $A$ \\
$c_{p B}$ & $128.464\left(\mathrm{JK}^{-1} \mathrm{~mol}^{-1}\right)$ & Heat capacity of species $B$ \\
$c_{p \text { Inert }}$ & $21.694\left(\mathrm{JK}^{-1} \mathrm{~mol}^{-1}\right)$ & Heat capacity of Inert \\
$E_{a}$ & $73.35\left(\mathrm{KJmol}^{-1}\right)$ & Activation energy \\
$h_{\text {Aref }}$ & $-5.808510^{5}\left(\mathrm{Jmol}^{-1}\right)$ & Reference enthalpy of $A$ \\
$h_{\text {Bref }}$ & $-6.688410^{5}\left(\mathrm{Jmol}^{-1}\right)$ & Reference enthalpy of $B$ \\
$h_{\text {Inertref }}$ & $-3.310^{5}\left(\mathrm{Jmol} \mathrm{J}^{-1}\right)$ & Reference enthalpy of Inert \\
$k_{0}$ & $2.5810^{9}\left(\mathrm{~s}^{-1}\right)$ & Kinetic constant \\
$R$ & $8.314\left(\mathrm{JK}^{-1} \mathrm{~mol}^{-1}\right)$ & Gas constant \\
$T_{\text {ref }}$ & $298(K)$ & Reference temperature \\
$\lambda$ & $0.75\left(\mathrm{WK}^{-1}\right)$ & Heat transfer coefficient \\
$d$ & $0.0070\left(\mathrm{~s}^{-1}\right)$ & Dilution rate \\
\hline
\end{tabular}

The exothermic reaction (55) is considered with $\nu_{A}=-1$ and $\nu_{B}=1$. The open and closed loop simulations are carried out with respect to two different initial conditions, $(\mathrm{C} 1)$ with $\left(T_{0}=340(K), N_{A 0}=0.04(\mathrm{~mol}), N_{B 0}=\right.$ $0.001(\mathrm{~mol}))$ and $(\mathrm{C} 2)$ with $\left(T_{0}=300(K), N_{A 0}=0.15(\mathrm{~mol}), N_{B 0}=\right.$ $0.03(\mathrm{~mol}))$.

\subsection{Open loop simulation}

Figure 1 shows that the system (56) has three steady states indicated with $P_{1}, P_{2}$ and $P_{3}$ under the input:

$$
\left\{\begin{array}{l}
T_{I}=T_{J}=298(\mathrm{~K}), N_{A I}=0.18(\mathrm{~mol}), \\
N_{B I}=0(\mathrm{~mol}), N_{\text {Inert } I}=3.57(\mathrm{~mol})
\end{array}\right.
$$

The intermediate steady state $P_{2}$ is unstable whereas $P_{1}$ and $P_{3}$ are (locally) stable. In the next subsection, we operate the reaction system at the unstable state $P_{2}$ using the feedback law defined by equation (34) for the jacket temperature $T_{J}$.

\subsection{Closed loop simulation}

In the first case we assume that all state variables are measured. In this case we can use the state feedback law (34). We choose $K_{T}=0.001, K_{A}=0$ 


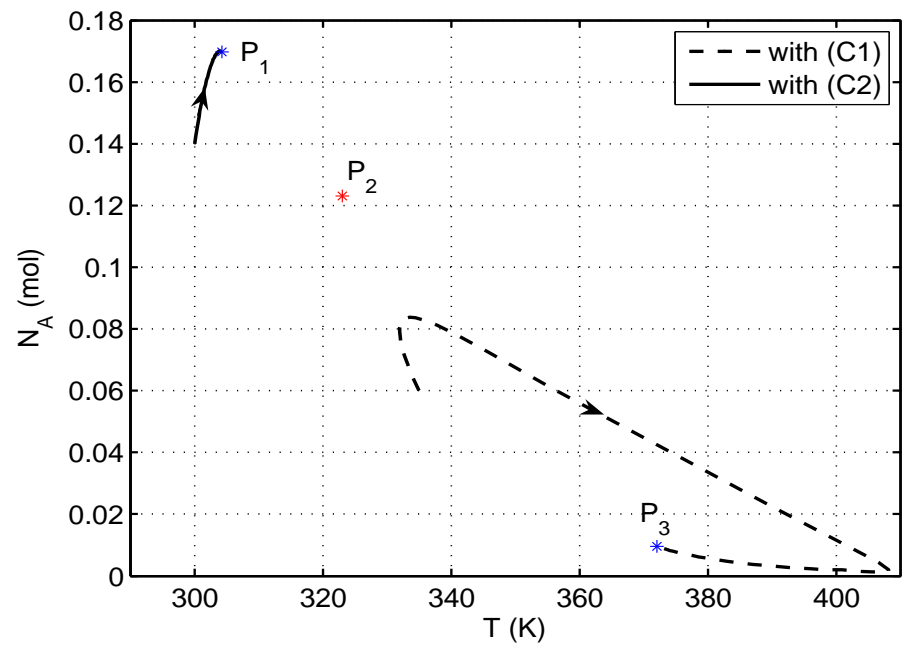

Figure 1: The representation of the open loop phase plane

and $K_{B}=0$ in the Hamiltonian function $\mathcal{H}_{d}(x)$ defined in equation (33). Figure 2 shows the closed loop response with phase plane. We see, for both of the considered initial conditions, that the system converges to the desired operating point $P_{2}$. Figure 2 also shows that the control variable input $T_{J}$ (34) is admissible in terms of amplitude and dynamics.

The Hamiltonian $\mathcal{H}_{d}(x)$ (33) plays the role of a global Lyapunov function for any choice of admissible initial conditions and consequently it converges to 0 as shown in Figure 3. 

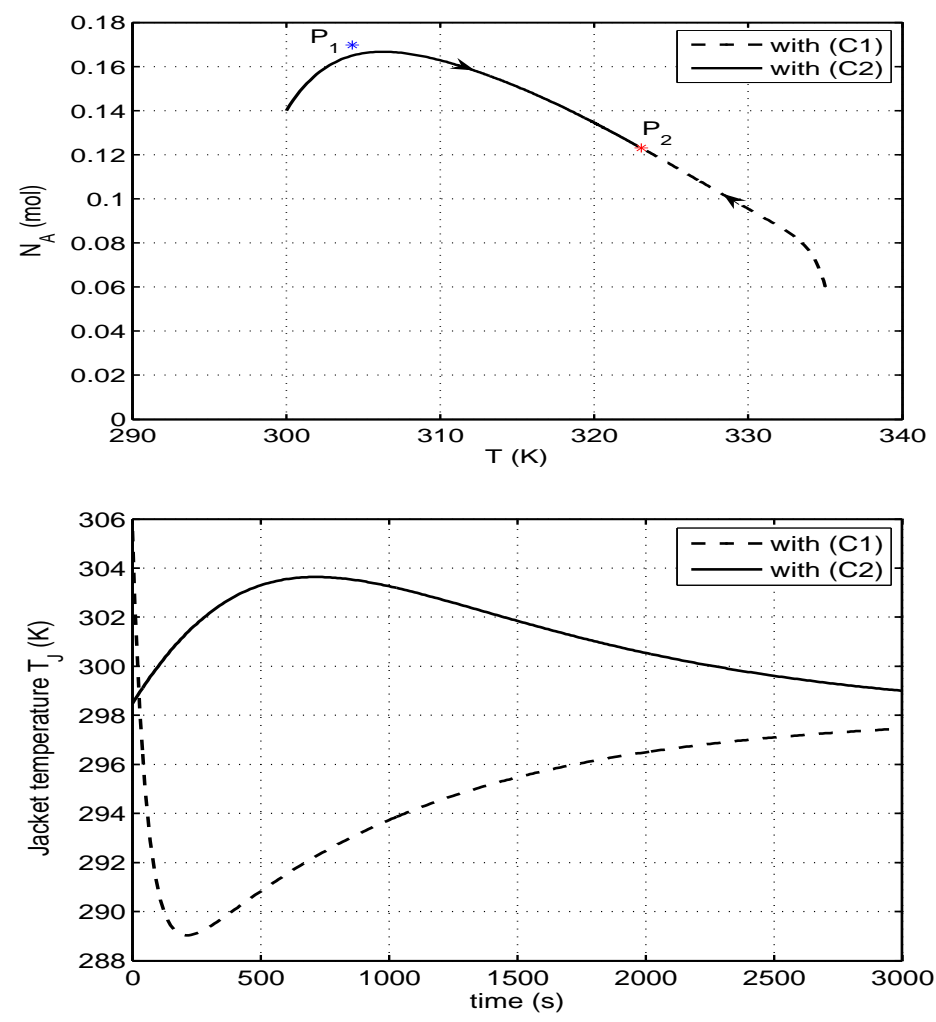

Figure 2: Representation of the closed loop phase plane (the point $P_{3}$ outside the frame) and the feedback law $T_{J}$ for two different initial conditions $(\mathrm{C} 1)$ and $(\mathrm{C} 2)$

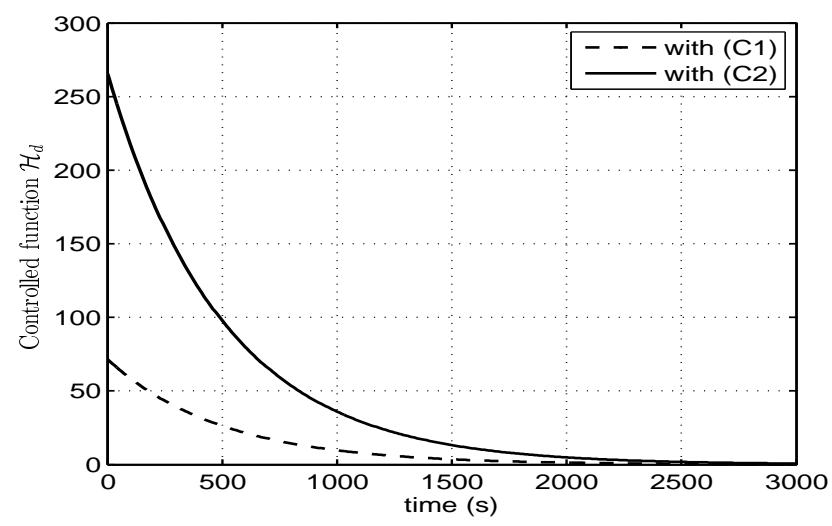

Figure 3: The dynamics of $\mathcal{H}_{d}$ 


\subsection{Simulation with the asymptotic observer}

First of all let us check that the feasibility conditions of Proposition 3 are satisfied. In our case, we have $\mathcal{I}=\emptyset, \mathcal{J}=\{A, B\}$ and $\boldsymbol{\nu}=\left(\begin{array}{c}\nu_{A} \\ \nu_{B}\end{array}\right)$. Thus $\left(\boldsymbol{\nu}^{\perp}\right)_{\mathcal{J}}=\left(\begin{array}{ll}\nu_{B} & -\nu_{A}\end{array}\right), \boldsymbol{h}_{\mathcal{J}}^{T}=\left(h_{A}(T) \quad h_{B}(T)\right)$ and $\operatorname{det}(\mathcal{O})=-\nu_{A} h_{A}(T)-$ $\nu_{B} h_{B}(T)=-\Delta H_{R}>0$. It follows that the observability matrix $\mathcal{O}$ is full rank and the asymptotic observer is feasible.

For the sake of simplicity, the initial condition $(\mathrm{C} 2)$ is used for the system. The initial conditions of the asymptotic observer are $(\hat{\mathrm{C}} 1)$ with $(\hat{H}(0)=$ $\left.0.98 H\left(T_{0}, N_{A 0}, N_{A 0}\right), \hat{N}(0)=0.75 N\left(N_{A 0}, N_{B 0}\right)\right)$ and $(\hat{\mathrm{C}} 2)$ with $(\hat{H}(0)=$ $\left.H\left(0.85 T_{0}, N_{A 0}, N_{B 0}\right), \hat{N}(0)=0.95 N\left(N_{A 0}, N_{B 0}\right)\right)$ where the numerical values of $T_{0}, N_{A 0}$ and $N_{B 0}$ are given with the initial condition (C2). The open loop convergence of the estimates generated by the asymptotic observer (51) is illustrated in Figure 4. With the initial condition (C2), the system converges to the stable point $P_{1}$. The closed loop simulations are given in Figures 5 and 6 with the initial conditions, $(\mathrm{C} 2)$ and $(\hat{\mathrm{C}} 1),(\mathrm{C} 2)$ and $(\hat{\mathrm{C}} 2)$ respectively. The stabilization at the unstable state $P_{2}$ of the controlled reaction system via the asymptotic observer is guaranteed. Furthermore, the dynamics of the control input $T_{J}$ remains admissible as seen in Figure 5(b) and Figure 6(b). 
(a)
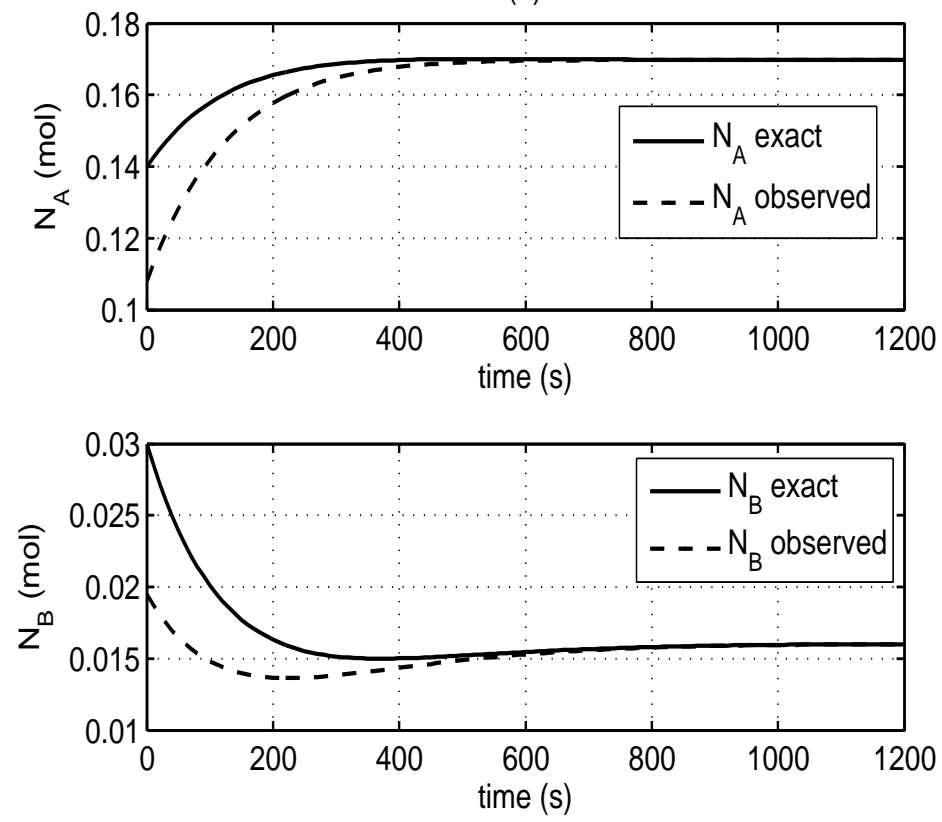

(b)
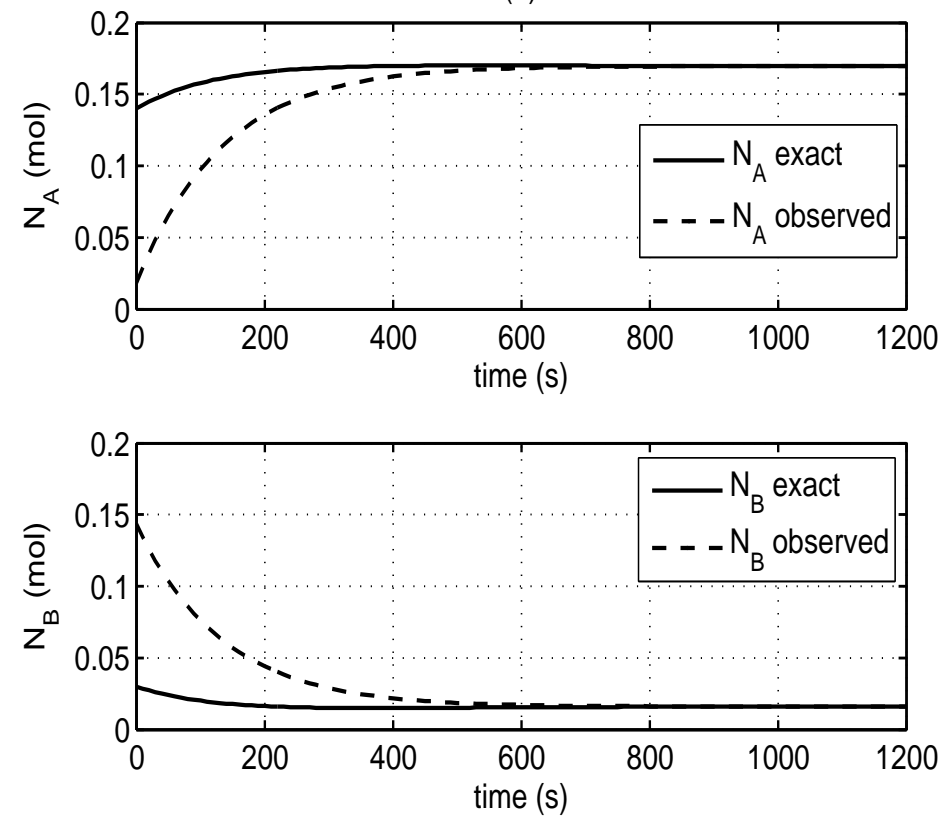

Figure 4: $N_{A}, N_{B}$ and their estimates in the open loop case -(a) for the initial conditions $(\mathrm{C} 2)$ and $(\hat{\mathrm{C}} 1)-(\mathrm{b})$ for initial conditions $(\mathrm{C} 2)$ and $(\hat{\mathrm{C}} 2)$ 
(a)
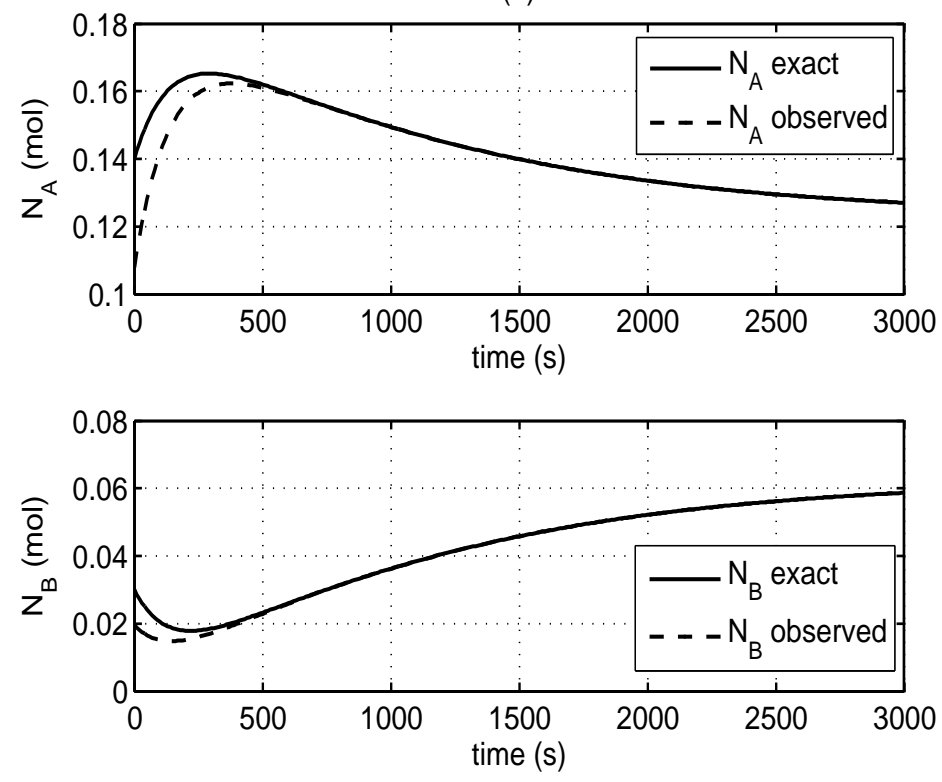

(b)

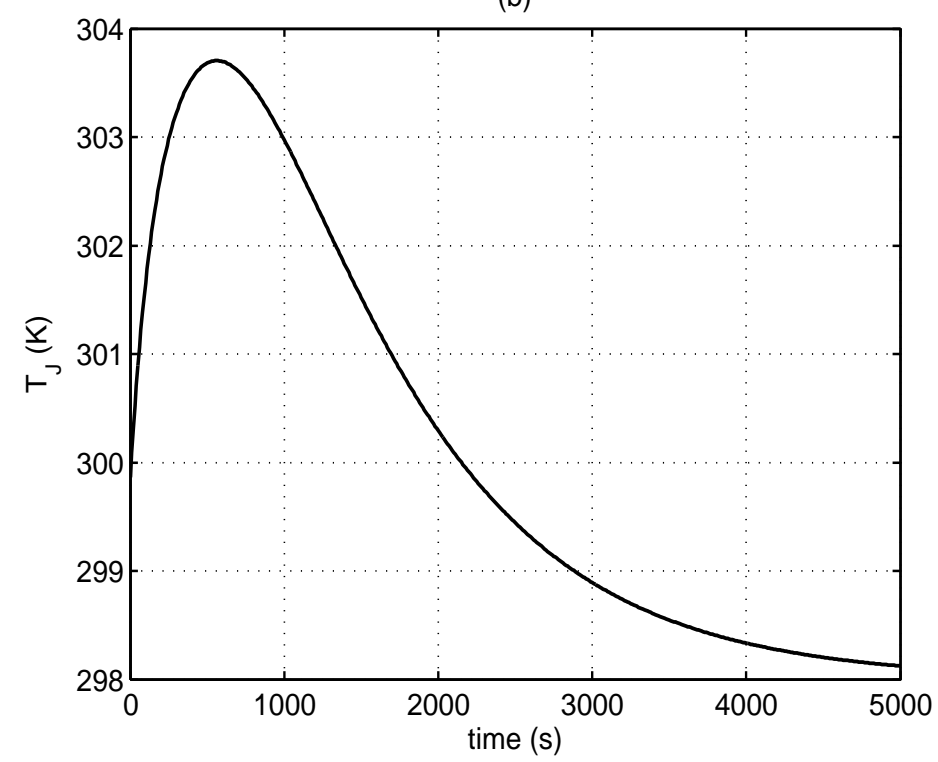

Figure 5: $N_{A}$ and its estimate with the asymptotic observer in the closed loop case -(a) for the initial conditions $(\mathrm{C} 2)$ and $(\hat{\mathrm{C}} 1)-(\mathrm{b})$ the control input $T_{J}$ with the asymptotic observer 
(a)
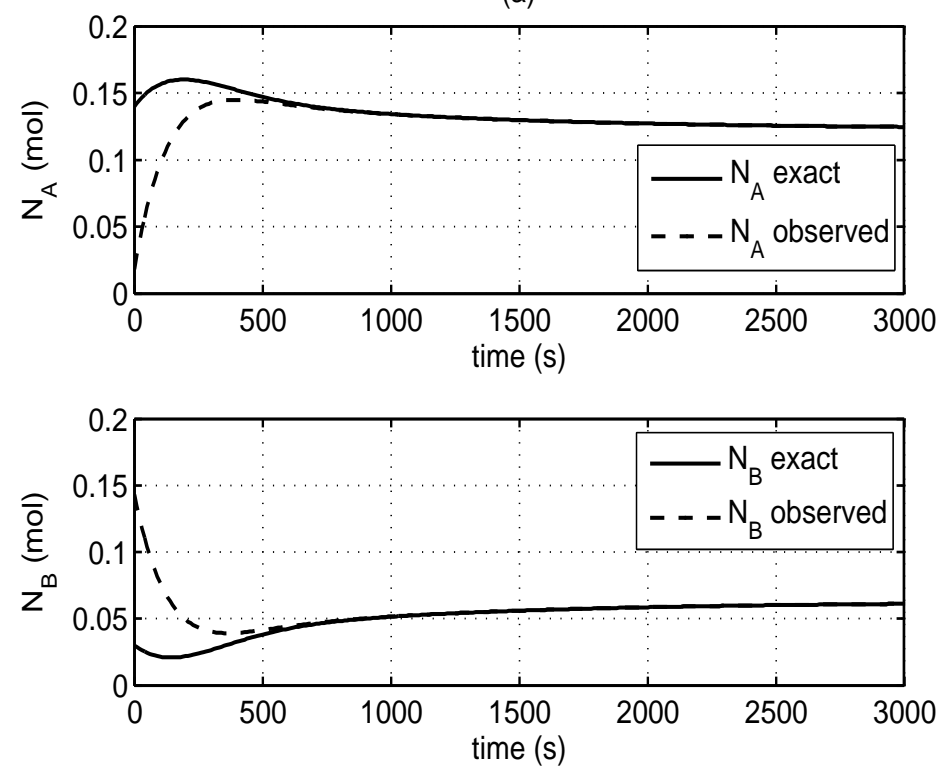

(b)

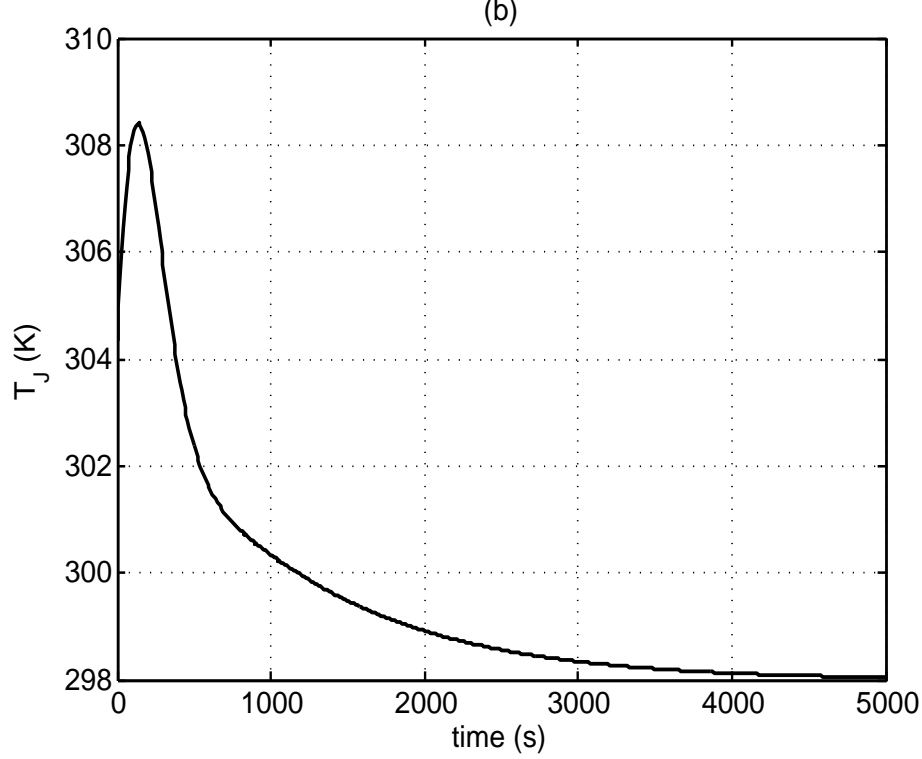

Figure 6: $N_{A}$ and its estimate with the asymptotic observer in the closed loop case -(a) for the initial conditions $(\mathrm{C} 2)$ and and $(\hat{\mathrm{C}} 2)-(\mathrm{b})$ the control input $T_{J}$ with the asymptotic observer 


\section{Conclusion}

We have shown, by means of the passivity-based approach in the port Hamiltonian framework, how to synthesize a nonlinear controller for the stabilization and how to design an asymptotic observer of a class of CSTRs. The results can be applied to non isothermal CSTRs operated under multiple steady states. The resulting state feedback developed in the paper generalizes the one proposed by [19] in the sense that we do not add a constraint on the control input. The closed loop convergence of the system is theoretically shown. The use of an asymptotic observer provided the rank condition on the observability matrix. This condition is fulfilled by appropriate choice of measured states. Finally, numerical simulations show that convergence objective is satisfied for a simple case study. The state feedback law on the jacket temperature $T_{J}$ is implementable and gives finite amplitude and admissible rate of variation. Open questions concern the structure of the observability matrix (with respect to traditional definition for linear systems); and the performance/robustness of the control law with respect to perturbations and parameters uncertainty.

\section{ACKNOWLEDGMENTS}

This work is supported by the Vietnam's National Foundation for Science and Technology Development (NAFOSTED) under research proposal number 101.01-2013.23. The first author wish to thank Prof. Denis Dochain for helpful discussions and comments on asymptotic observers during his stay in CESAME, UCL, Belgium. The scientific responsibility rests with its authors.

\section{References}

[1] H. K. Khalil, Nonlinear systems, 2rd edition, Prentice Hall, 2002.

[2] J. C. Willems, Dissipative dynamical systems. Part I: General theory, Arch. Rat. Mech. and Analysis. 45(5):321-351, 1972.

[3] A. van der Schaft, $L_{2}$-gain and passivity techniques in nonlinear control, Springer-Verlag, London, 2nd edt, 2000.

[4] B. Brogliato, R. Lozano, B. Maschke and O. Egeland, Dissipative systems analysis and control, Springer, London, 2nd edition, 2007. 
[5] R. Ortega, A. van der schaft, I. Mareels and B. Maschke, Putting energy back in control, IEEE Control Systems Magazine. 21(2):18-33, 2001.

[6] R. Ortega, D. Jeltsema and J.M.A. Scherpen, Power shaping: A new paradigm for stabilization of nonlinear $R L C$ circuits, IEEE Trans. on Autom. Control. 48(10):1762-1767, 2003.

[7] D. Jeltsema, R. Ortega, and J.M.A. Scherpen, An energy-balancing perspective of interconnection and damping assignment control of nonlinear systems, Automatica. 40(9):1643-1646, 2004.

[8] R. Ortega, A. van der Schaft, B. Maschke and G. Escobar, Interconnection and damping assignment passivity-based control of port-controlled Hamiltonian Systems, Automatica. 38:585-596, 2002.

[9] A. van der Schaft, Port-controlled Hamiltonian systems: Towards a theory for control and design of nonlinear physical systems, SICE Journal. 39(2):91-98, 2000.

[10] B. Maschke, R. Ortega and A. van der Schaft, Energy based Lyapunov functions for forced Hamiltonian systems with dissipation, IEEE Trans. on Autom. Control. 45(8):1498-1502, 2000.

[11] J. Alvarez, J. Alvarez-Ramírez, G. Espinosa-Perez and A. Schaum, Energy shaping plus damping injection control for a class of chemical reactors, Chem. Eng. Sci. 66(23):6280-6286, 2011.

[12] A. Favache and D. Dochain, Power-shaping of reaction systems : the CSTR case study, Automatica. 46(11):1877-1883, 2010.

[13] K.M. Hangos, J. Bokor and G. Szederkényi, Hamiltonian view on process systems, AIChE journal. 47(8):1819-1831, 2001.

[14] H. Ramírez, D. Sbarbaro and R. Ortega, On the control of non-linear processes: An IDA-PBC approach, J. Proc. Control. 19:405-414, 2009.

[15] H. Hoang, F. Couenne, C. Jallut and Y. Le Gorrec, Lyapunov-based control of non isothermal continuous stirred tank reactors using irreversible thermodynamics, J. Proc. Control. 22(2):412-422, 2012. 
[16] N. Hudon and J. Bao, Dissipativity-based decentralized control of interconnected nonlinear chemical processes, Computers $\& 3$ Chemical Engineering. 45(12):84-101, 2012.

[17] W.L. Luyben, Process modeling, simulation, and control for chemical engineers, McGraw-Hill, Singapore, 1990.

[18] M.P. Niemiec and C. Kravaris, Nonlinear model-state feedback control for a nonminimum-phase processes. Automatica. 39:1295-1302, 2003.

[19] F. Viel, F. Jadot and G. Bastin, Global stabilization of exothermic chemical reactors under input constraints, Automatica. 33(8):1437-1448, 1997.

[20] C. Georgakis, On the use of extensive variables in process dynamics and control, Chem. Eng. Sci. 41(6):1471-1484, 1986.

[21] H. Hoang, F. Couenne, C. Jallut and Y. Le Gorrec, The Port Hamiltonian approach to modeling and control of Continuous Stirred Tank Reactors, J. Proc. Control. 21(10):1449-1458, 2011.

[22] B. Srinivasan, M. Amrhein, and D. Bonvin, Reaction and Flow Variants/Invariants in Chemical Reaction Systems with Inlet and Outlet Streams, AIChE journal. 44(8):1855-1867, 1998.

[23] A. Favache, D. Dochain and J. Winkin, Power-shaping control: writing the system dynamics into the Brayton-Moser form, Syst. Cont. Let. 60(8): 618-624, 2011.

[24] A.M. Gibon-Fargeot, H. Hammouri and F. Celle, Nonlinear observers for chemical reactors, Chem. Eng. Sci. 49(14):2287-2300, 1994.

[25] M. Soroush, Nonlinear state-observer design with application to reactors, Chem. Eng. Sci. 52(3):387-404, 1997.

[26] J. Alvarez-Ramírez, Observers for a class of continuous tank reactors via temperature measurement, Chem. Eng. Sci. 55(9):1393-1399, 1995.

[27] D. Dochain, F. Couenne and C. Jallut, Enthalpy based modelling and design of asymptotic observers for chemical reactors, International Journal of Control. 82(8):1389-1403, 2009. 
[28] D.D. Bruns and J.E. Bailey, Process operation near an unstable steady state using nonlinear feedback control, Chem. Eng. Sci. 30:755-762, 1975 .

[29] J. Alvarez-Ramírez and A. Morales, PI control of continuously stirred tank reactors: stability and performance, Chem. Eng. Sci. 55(22):54975507, 2000.

[30] R. Antonelli and A. Astolfi, Continuous stirred tank reactors: easy to stabilise?, Automatica. 39:1817-1827, 2003.

[31] F. Dörfler, J.K. Johnsen and F. Allgöwer, An introduction to interconnection and damping assignment passivity-based control in process engineering, J. Proc. Control. 19:1413-1426, 2009.

[32] C.A. Farschman, K.P. Viswanath and B.E. Ydstie, Process systems and inventory control, AIChE journal. 44(8):1841-1857, 1998.

[33] N. Hudon, K. Höffner and M. Guay, Equivalence to dissipative Hamiltonian realization, Proc. 47 th IEEE Conference on Decision and Control. 3163-3168, 2008.

[34] B.E. Ydstie and A.A. Alonso, Process systems and passivity via the Clausius-Planck inequality, Syst. ES Contr. Let. 30(5):253-264, 1997.

[35] D. Dochain, State and parameter estimation in chemical and biochemical processes: A tutorial. J. Proc. Control. 13:801-818, 2003.

[36] C. Kravaris, J. Hahn and Y. Chu, Advances and selected recent developments in state and parameter estimation, Computers $\&$ Chemical Engineering, http://dx.doi.org/10.1016/j.compchemeng.2012.06.001, 2012.

[37] D. Dochain, M. Perrier and B.E. Ydstie, Asymptotic observers for stirred tank reactors, Chem. Eng. Sci. 47(15-16):4167-4117, 1992.

[38] F. Couenne, C. Jallut, B. Maschke, P.C. Breedveld and M. Tayakout, Bond graph modelling for chemical reactors, Mathematical and Computer Modelling of Dynamical Systems. 12(2-3):159-174, 2006.

[39] J.G. van de Vusse, Plug-Flow Type Reactor Versus Tank Reactor, Chem. Eng. Sci. 19:994-998, 1964. 
[40] J.A. Moreno and D. Dochain, Global observability and detectability analysis of uncertain reaction systems and observer design, International Journal of Control. 81(7):1062-1070, 2007.

[41] H. Hoang, F. Couenne, Y. Le Gorrec, C.L. Chen and B.E. Ydstie, Passivity based controller and observer for exothermic chemical reactors. IFAC-ADCHEM, July 10-13, Singapore. pp. 377-384, 2012. 